\title{
Remarkable electronic effect on the total stereoselectivity of the cycloaddition reaction of arylnitrile oxides with pyrrol-2-one derivatives
}

https://doi.org/10.1515/hc-2019-0014

Received December 11, 2018; accepted March 14, 2019.

Abstract: The regiospecific 1,3-dipolar cycloaddition of 1,5-dihydropyrrol-2-one and arylnitrile oxides derivatives have been investigated. The asymmetric induction expected by the chiral centre of the 5-hydroxy-3-methyl-1,5dihydropyrrol-2-one derivatives was very effective, single diastereoisomers anti-3 was formed. The diastereoselectivity was linked to the destabilization of the syn transition state as a result of the electrostatic repulsion between the hydroxy group of the dihydropyrrol-2-one derivatives and the atom oxygen of the dipole.

Keywords: Cycloaddition 1,3-dipolar, 1,5-dihydropyrrol-2one, arylnitrile oxides, regiospecifically, diastereoisomers.

\section{Introduction}

1,3-Dipolar cycloaddition of nitrile oxides to alkenes is the most useful method of preparation for isoxazolines which can be easily converted to several synthetically important compounds such as $\beta$-hydroxy ketones, $\beta$-hydroxy esters, $\alpha, \beta$-unsaturated carbonyl compounds [1-3]. The regio- and stereoselectivity of these reactions dramatically depends on the nature of the substituents

\footnotetext{
* Corresponding author: N Ben Hamadi, Chemistry Department, College of Science, IMSIU (Al Imam Mohammad Ibn Saud Islamic University), Riyadh 11623, kingdom of Saudi Arabia; Laboratory of Synthesis Heterocyclic and Natural Substances, Faculty of Sciences of Monastir, UM (University of Monastir), Boulevard of Environment, 5000 Monastir, Tunisia. e-mail: bh_naoufel@yahoo.fr, Tel.: 0021652557560 , Fax: 0021673500278.

A Guesmi, Textile Engineering Laboratory, Higher Institute of Technological Studies of Ksar Hellal, UM (University of Monastir), Tunisia; Chemistry Department, College of Science, IMSIU (Al Imam Mohammad Ibn Saud Islamic University), Riyadh 11623, kingdom of Saudi Arabia.
}

on both the alkene and dipole $[4,6]$. The isoxazoline ring is present in a number of commercially available and clinically useful drugs, as well as in other biologically active compounds, acting as antibacterial, antitubercular, and antidepressant agents $[7,8]$. Most of the recently synthesized isoxazolines were prepared as antibacterial and antifungal compounds [9]. Based on an evaluation of the nitrile oxides cycloaddition, it was felt that the stereochemistry of these new centers could be controlled if the reaction system were properly designed. Pyrrolidinones are important precursors in organic synthesis and are found in many pharmaceuticals and active natural products $[10,11]$.

As a continuation of our effort to utilize heterocyclic compounds as dipolarophiles in 1,3-dipolar cycloaddition reactions, we report the asymmetric 1,3-cycloaddition of aromatic nitrile oxides with pyrrol-2-one derivatives [12-14].

\section{Results and discussions}

The labile nitrile oxides 2a-c generated in situ were allowed to react with pyrrolidinones 1a,b in toluene [15]. The reaction of 1,5-dihydropyrrol-2-one derivatives $\mathbf{1}$ and the arylnitrile oxides $2[5,6]$ proceeded with the formation of single diastereoisomers 3 (Scheme 1).

We now have to determine the addition mode of arylnitrile oxides with 1. Unambiguous proofs for the obtained cycloadducts regiochemistry arose from their spectral data. However, regiochemical assignments of all adduct were deduced from their ${ }^{13} \mathrm{C}$-NMR spectra. In particular, the chemical shifts of C-6a are in excellent agreement with those usually obtained when this quaternary carbon is attached to oxygen atom [16].

The attack of the 1,3-dipole occurred from the less hindered face of the dipolarophile 1 giving the single isomer 3. The electrostatic repulsion should account for 


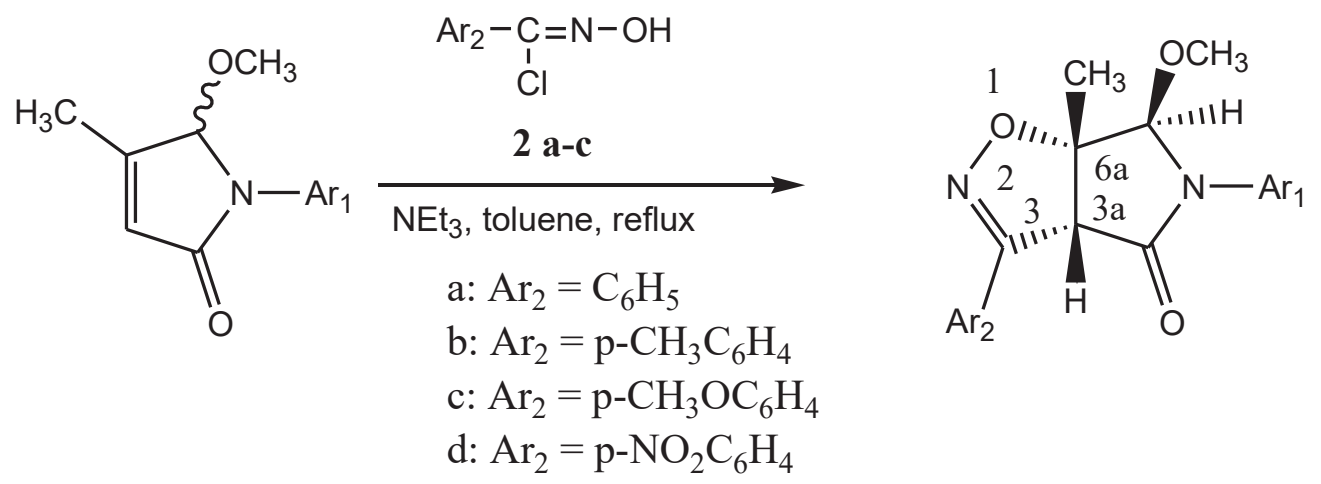

1a,b

Anti-3

a: $\mathrm{Ar}_{1}=\mathrm{C}_{6} \mathrm{H}_{5}$

b: $\mathrm{Ar}_{1}=\mathrm{p}-\mathrm{CH}_{3} \mathrm{OC}_{6} \mathrm{H}_{4}$

\section{Cycloadducts}

a: $\mathrm{Ar}_{1}=\mathrm{C}_{6} \mathrm{H}_{5}, \mathrm{Ar}_{2}=\mathrm{C}_{6} \mathrm{H}_{5}$

b: $\mathrm{Ar}_{1}=\mathrm{C}_{6} \mathrm{H}_{5}, \mathrm{Ar}_{2}=\mathrm{p}-\mathrm{CH}_{3} \mathrm{C}_{6} \mathrm{H}_{4}$

c: $\mathrm{Ar}_{1}=\mathrm{C}_{6} \mathrm{H}_{5}, \mathrm{Ar}_{2}=\mathrm{p}-\mathrm{CH}_{3} \mathrm{OC}_{6} \mathrm{H}_{4}$

d: $\mathrm{Ar}_{1}=\mathrm{C}_{6} \mathrm{H}_{5}, \mathrm{Ar}_{2}=\mathrm{p}-\mathrm{NO}_{2} \mathrm{C}_{6} \mathrm{H}_{4}$

e: $\mathrm{Ar}_{1}=\mathrm{p}-\mathrm{CH}_{3} \mathrm{OC}_{6} \mathrm{H}_{4}, \mathrm{Ar}_{2}=\mathrm{C}_{6} \mathrm{H}_{5}$

$\mathrm{f}: \mathrm{Ar}_{1}=\mathrm{p}-\mathrm{CH}_{3} \mathrm{OC}_{6} \mathrm{H}_{4}, \mathrm{Ar}_{2}=\mathrm{p}-\mathrm{CH}_{3} \mathrm{C}_{6} \mathrm{H}_{4}$

g: $\mathrm{Ar}_{1}=\mathrm{p}-\mathrm{CH}_{3} \mathrm{OC}_{6} \mathrm{H}_{4}, \mathrm{Ar}_{2}=\mathrm{p}-\mathrm{CH}_{3} \mathrm{OC}_{6} \mathrm{H}_{4}$

h: $\mathrm{Ar}_{1}=\mathrm{p}-\mathrm{CH}_{3} \mathrm{OC}_{6} \mathrm{H}_{4}, \mathrm{Ar}_{2}=\mathrm{p}-\mathrm{NO}_{2} \mathrm{C}_{6} \mathrm{H}_{4}$
Yield (\%)

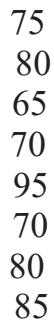

80

65

70

95

70

80

85

Scheme 1 Cycloaddition 1,3-dipolar of nitrile oxides with pyrrole-2-one derivatives.

the observed results. Moreover, syn orientation of the oxygen-containing pyrrolidinones to the oxygen atom of nitrile oxide leads to greater repulsion in the transition state (Figure 1) [17,18].

The anti stereochemistry of the 2-isoxazolines $\mathbf{3}$ was deduced from the NOES spectrum which allowed us to distinguish a clear spatial correlation between the $\mathrm{CH}_{3}$ protons (5.4 ppm), H-3a (4.14) and the methoxy proton (3.8 $\mathrm{ppm})$. Also, the absence of any nOe between the methoxy H-6 proton (6.9 ppm) and the methyl protons (1.6 ppm) of the dipolarophile moiety, confirms the structure of the obtained compounds 3 (Figure 2).

\section{Conclusion}

Our studies have shown that methoxy group is extremely effective in affording complete stereoselectivity for 1,3dipolar cycloaddition reactions between nitrile oxides and pyrrolidinones. This synthesis allows us to obtain one diastereoisomer of isoxazolines. Finally, the electrostatic repulsion between oxygen-containing pyrrolidinones and the oxygen atom of nitrile oxide are the main reasons for the observed anti-selectivity.

\section{Experimental details}

\section{General}

Infrared spectra were recorded on a Perkin-Elmer IR-197 spectrophotometer in $\mathrm{KBr}$ disks. NMR spectra were obtained with a Bruker AC 300 spectrometer operating at 300 $\mathrm{MHz}$ for $1 \mathrm{H}$ and at $75.64 \mathrm{MHz}$ for $13 \mathrm{C}$ using TMS as the internal standard. Elemental analysis was performed with a Perkin-Elmer 240B microanalyzer. The melting points, thermal transitions, and mesomorphic textures were determined using an Olympus BX50 microscope equipped with a Mettler Toledo FP-82 hot-stage and a PM-30 exposure control unit.

\section{Materials}

All the reagents were obtained from commercial sources and used without further purification. The organic solvents were of commercial grade quality and all were dried by traditional methods. In general, all the compounds were purified by column chromatography on silica gel (60-120 mesh), and crystallization from analytical grade 
<smiles>CO[C@H]1C(C)=CC(=O)N1Br</smiles><smiles>[C+]C=N[O]</smiles>

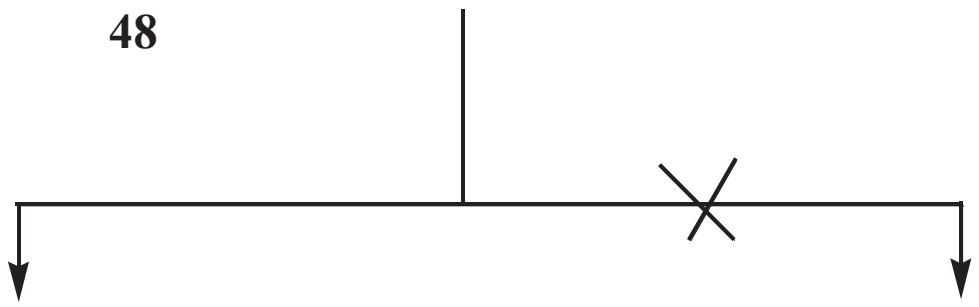

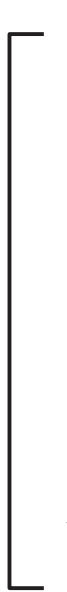

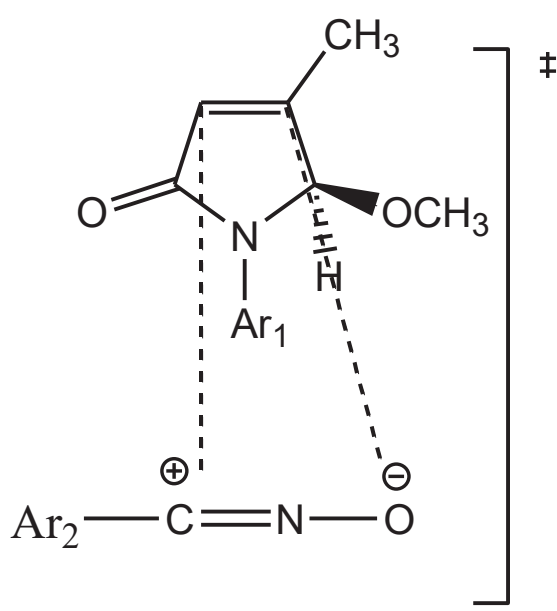

TS-anti

$\downarrow$<smiles>[Y17]C1=NO[C@@H]2C(=O)[C@@H](OC)[C@H](C)[C@@H]12</smiles>

Anti-3

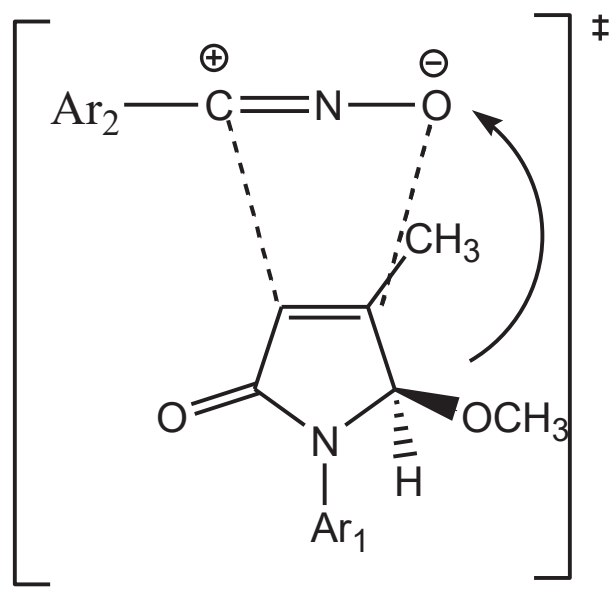

TS-syn
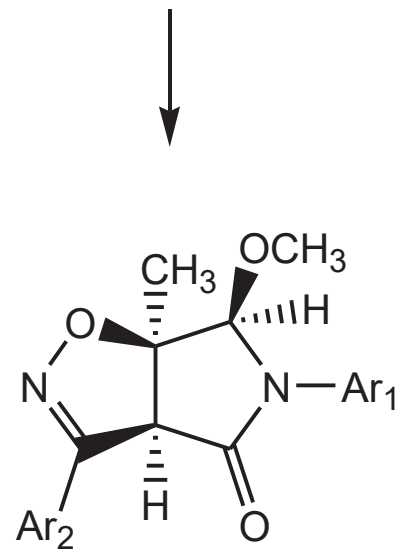

Syn-3

Figure 1 Stereochemistry of the 2-isoxazolines 3. 
solvents. The purity of the sample was checked by thinlayer chromatography (Merck Kieselgel 60F254).

\section{Addition of aromatic nitrile oxides to dihydropyrrol- 2-one derivatives}

A solution of dipolarophiles 1a-b (1 mmol) and chloroximes $2 \mathbf{c}-\mathbf{e}(1.1 \mathrm{mmol})$ in toluene $(10 \mathrm{~mL})$, was stirred at 110 C. To this solution trimethylamine $(0.2 \mathrm{~mL})$, dissolved in toluene $(10 \mathrm{~mL})$, was added dropwise. The precipitated triethylammonium chloride was removed by filtration and the filtrate was concentrated in vacuo, and chromatography $\left(\mathrm{SiO}_{2}\right.$; ethyl acetate/petroleum ether, 2:1) to afford compounds 3a-f.

\section{6-Methoxy-6a-methyl-3,5-diphenyl-3a,5,6,6a- tetrahydro-4H-pyrrolo[3,4-d]isoxazole-4-one (3a)}

Yield $=75 \%$ white solid. $\mathrm{mp}=221-222{ }^{\circ} \mathrm{C}$. IR $(\mathrm{KBr})$ ymax $/$ $\mathrm{cm}^{-1}: 1645(\mathrm{C}=\mathrm{N}) ; 1735(\mathrm{C}=0) .{ }^{1} \mathrm{H}$ NMR $(300 \mathrm{MHz}$; DMSOd6) $\delta_{\mathrm{ppm}}: 1.61\left(\mathrm{~s}, 3 \mathrm{H}, \mathrm{CH}_{3}\right) ; 3,80\left(\mathrm{~s}, 3 \mathrm{H}, \mathrm{OCH}_{3}\right) ; 4.12(\mathrm{~s}, 1 \mathrm{H}$, 3a-H); 6.47 (s, 1H, 6-H); 7.27-7.87 (m, 10H, Harom). ${ }^{13} \mathrm{C}$ NMR (75.47 MHz; DMSO-d6) $\delta_{\mathrm{ppm}}: 19.5 ;$ 55.5; 61.5; 89.5; 91.4;
122.6-137.0; 153.8; 170.1. Elemental analysis: $\mathrm{C}_{19} \mathrm{H}_{18} \mathrm{~N}_{2} \mathrm{O}_{3}$ requires $\mathrm{C}, 70.79$; $\mathrm{H}, 5.63$; N, 8.69\%; Found: $\mathrm{C}, 70.81 ; \mathrm{H}$, 5.60; N, 8.73\%.

\section{6-Methoxy-6a-methyl-3-(4-methylphenyl)-5-phenyl- 3a,5,6,6a-tetrahydro-4Hpyrrolo[3,4-d]isoxazole-4-one (3b)}

Yield $=80 \%$ white solid. $\mathrm{mp}=191-192{ }^{\circ} \mathrm{C}$. IR $(\mathrm{KBr})$ ymax $/$ $\mathrm{cm}^{-1}: 1635(\mathrm{C}=\mathrm{N})$; $1735(\mathrm{C}=0) .{ }^{1} \mathrm{H}$ NMR $(300 \mathrm{MHz}$; DMSOd6) $\delta_{\mathrm{ppm}}: 1.68\left(\mathrm{~s}, 3 \mathrm{H}, \mathrm{CH}_{3}\right) ; 2.54\left(\mathrm{~s}, 3 \mathrm{H}, \mathrm{CH}_{3}\right) ; 3.77(\mathrm{~s}, 3 \mathrm{H}$, $\left.\mathrm{OCH}_{3}\right) ; 4.21$ (s, 1H, 3a-H); 6,61 (s, 1H, 6-H); 7,36-7,80 (m, 9H, Harom). ${ }^{13} \mathrm{C}$ NMR (75.47 MHz; DMSO-d6) $\delta_{\mathrm{ppm}}: 19.1 ; 21.0 ; 55.8$; 60.2; 89.5; 91.1; 123.4-141.9; 154.3; 168.9. Elemental analysis: $\mathrm{C}_{20} \mathrm{H}_{20} \mathrm{~N}_{2} \mathrm{O}_{3}$ requires $\mathrm{C}, 71.41 ; \mathrm{H}, 5.99 ; \mathrm{N}, 8.33 \%$; Found: C, 71.46; H, 5.96; N, 8.34\%.

\section{6-Methoxy-3-(4-methoxyphenyl)-6a-methyl-5-phenyl- 3a,5,6,6a-tetrahydro-4H-pyrrolo[3,4-d]isoxazole-4-one (3c)}

Yield $=65 \%$ white solid. $\mathrm{mp}=199-200^{\circ} \mathrm{C}$. IR $(\mathrm{KBr})$ ymax $/$ $\mathrm{cm}^{-1}$ : $1630(\mathrm{C}=\mathrm{N}) ; 1745(\mathrm{C}=\mathrm{O}) .{ }^{1} \mathrm{H}$ NMR (300 MHz; DMSO) $\delta_{\mathrm{ppm}}: 1.68\left(\mathrm{~s}, 3 \mathrm{H}, \mathrm{CH}_{3}\right) ; 3.81\left(\mathrm{~s}, 3 \mathrm{H}, \mathrm{OCH}_{3}\right) ; 3.82(\mathrm{~s}, 3 \mathrm{H}$, $\left.\mathrm{OCH}_{3}\right) ; 4.18$ (s, 1H, 3a-H); $6.53(\mathrm{~s}, 1 \mathrm{H}, 6-\mathrm{H}) ;$ 7.17-7.87 (m, 9H,
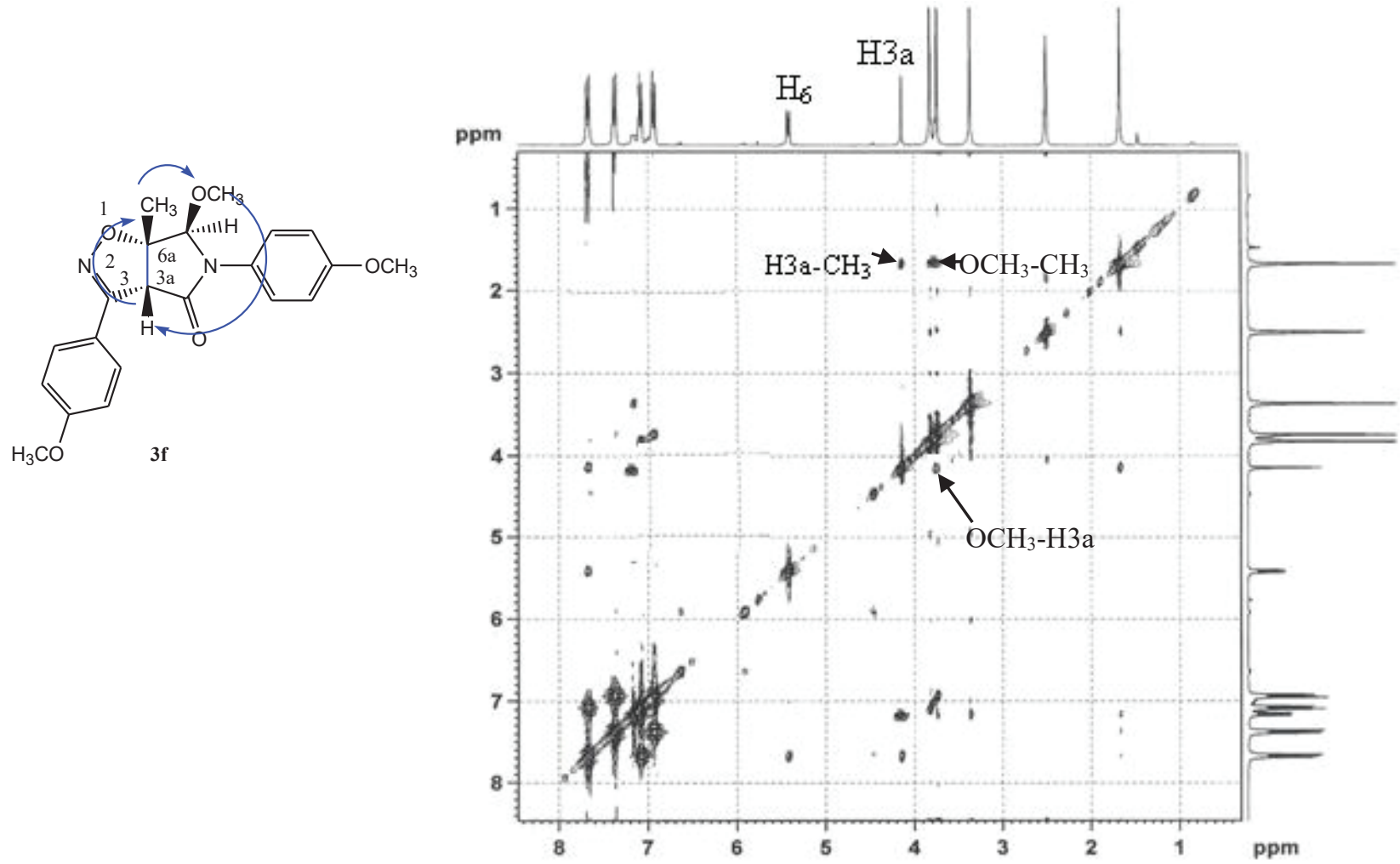

Figure 2 NOESY spectrum of isoxazoline $\mathbf{3 f}$. 
Harom). ${ }^{13} \mathrm{C}$ NMR (75,47 MHz; DMSO) $\delta_{\mathrm{ppm}}: 20.2 ;$ 55.7; 55.9; 60.4; 85.4; 89.3; 113.9-162.4; 157.2; 171.5.

Elemental analysis: $\mathrm{C}_{20} \mathrm{H}_{20} \mathrm{~N}_{2} \mathrm{O}_{4}$ requires $\mathrm{C}, 68.17 ; \mathrm{H}, 5.72 ; \mathrm{N}$, 7.95\%; Found: C, 68.20; H, 5.69; N, 7.99\%.

\section{6-Methoxy-6a-methyl-3-(4-nitro-phenyl)-5-phenyl- 3a,5,6,6a-tetrahydro-pyrrolo[3,4-d]isoxazol-4-one (3d)}

Yield $=70 \%$ white solid. $\mathrm{mp}=201-202^{\circ} \mathrm{C}$. IR $(\mathrm{KBr}) \mathrm{ymax} / \mathrm{cm}^{-1}$ : $1635(\mathrm{C}=\mathrm{N}) ; 1740(\mathrm{C}=\mathrm{O}) .{ }^{1} \mathrm{H}$ NMR (300 MHz; DMSO) $\delta_{\mathrm{ppm}}: 1.66$ (s, 3H, $\left.\mathrm{CH}_{3}\right) ; 3.80$ (s, 3H, OCH $)$; 4.21 (s, $\left.1 \mathrm{H}, 3 \mathrm{a}-\mathrm{H}\right) ; 6.48$ (s, $1 \mathrm{H}$, 6-H); 7.17-8.11 (m, 9H, Harom). ${ }^{13} \mathrm{C}$ NMR (75,47 MHz; DMSO) $\delta_{\mathrm{ppm}}: 20.1 ; 55.8 ; 60.5 ; 85.1 ; 88.9 ; 116.49-149.4 ; 157.4 ; 170.9$.

Elemental analysis: $\mathrm{C}_{19} \mathrm{H}_{17} \mathrm{~N}_{3} \mathrm{O}_{5}$ requires $\mathrm{C}, 62.12 ; \mathrm{H}, 4.66 ; \mathrm{N}$, 11.44\%; Found: C, 62.14; H, 4.59; N, 11.38\%.

\section{6-Methoxy-5-(4-methoxyphenyl)-6a-methyl-3-phenyl- $3 a, 5,6,6$ atetrahydro- $4 \mathrm{H}$ pyrrolo[3,4- d]isoxazole-4-one (3e)}

Yield $=95 \%$ white solid. $\mathrm{mp}=178-179{ }^{\circ} \mathrm{C} . \mathrm{IR}(\mathrm{KBr}) \mathrm{ymax} /$ $\mathrm{cm}^{-1}$ : $1635(\mathrm{C}=\mathrm{N})$; $1737(\mathrm{C}=\mathrm{O}) .{ }^{1} \mathrm{H}$ NMR (300 MHz; DMSOd6) $\delta_{\mathrm{ppm}}: 1.43\left(\mathrm{~s}, 3 \mathrm{H}, \mathrm{CH}_{3}\right) ; 3.80\left(\mathrm{~s}, 3 \mathrm{H}, \mathrm{OCH}_{3}\right) ; 3.81(\mathrm{~s}, 3 \mathrm{H}$, $\left.\mathrm{OCH}_{3}\right) ; 4.67$ (s, 1H, 3a-H); 6.94 (s, 1H, 6-H); 7.02 (d, 2H, J = $9 \mathrm{~Hz})$ and $7.80(\mathrm{~d}, 2 \mathrm{H}, J=9 \mathrm{~Hz}):$; 7.22-7.55 (m, 5H, Harom). ${ }^{13} \mathrm{C}$ NMR (300 MHz; DMSO-d6) $\delta_{\mathrm{ppm}}$ : 19.2; 55.7; 55.60; 59.4; 88.8; 91.9; 114.6-162.7; 156.3; 168.7. Elemental analysis: $\mathrm{C}_{20} \mathrm{H}_{20} \mathrm{~N}_{2} \mathrm{O}_{4}$ requires C, 68.17; $\mathrm{H}, 5.72 ; \mathrm{N}, 7.95 \%$; Found: C, 68.15; H, 5.65; N, 8.03\%.

\section{6-Methoxy-5-(4-methoxyphenyl)-6a-methyl-3-(4- methylphenyl)-3a,5,6,6a-tetrahydro-4H-pyrrolo[3,4-d] isoxazole-4-one ( $3 f$ )}

Yield $=70 \%$ white solid. $\mathrm{mp}=168-169^{\circ} \mathrm{C} . \mathrm{IR}(\mathrm{KBr}) \mathrm{ymax} /$ $\mathrm{cm}^{-1}: 1640(\mathrm{C}=\mathrm{N}) ; 1745(\mathrm{C}=\mathrm{O}) .{ }^{1} \mathrm{H}$ NMR (300 MHz; DMSO-d6)

\section{References}

[1] K. Al-Duaij, O.; Ben Hamadi, N.; Khezemi, L. Asymmetric Cycloaddition: An Efficient Synthesis of Enantiopure Isoxazolines Substituted with Carbohydrate Analogues. Journal of Heterocyclic Chemistry, 2016, 53, 408-413.

[2] Ben Hamadi, N.; Msaddek, M. Synthesis and evolution of new isoxazolo-1,4-quinones of biologic interest. Heterocyclic Communications, 2006, 12, 457-462.

[3] Jeffrey, W.B.; Erick, M.C. A Mild and Chemoselective Method for the Reduction of Conjugated Isoxazolines to $\beta$-Hydroxy Ketones. Org. Lett., 2001, 3, 1587-1590. $\delta_{\mathrm{ppm}}: 1.46\left(\mathrm{~s}, 3 \mathrm{H}, \mathrm{CH}_{3}\right) ; 2.39$ (s, 3H, $\left.\mathrm{CH}_{3}\right) ; 3.81$ (s, 3H, $\left.\mathrm{OCH}_{3}\right)$; 3.82 (s, 3H, $\left.\mathrm{OCH}_{3}\right) ; 4.65$ (s, 1H, 3a-H); 6.91 (s, 1H, 6- H); 6.96 $(\mathrm{d}, 2 \mathrm{H}, J=9 \mathrm{~Hz})$ and $7.43(\mathrm{~d}, 2 \mathrm{H}, J=9 \mathrm{~Hz}) ; 7.27(\mathrm{~d}, 2 \mathrm{H}, J=$ $7.8 \mathrm{~Hz}$ ) and 7.83 (d, 2H, $J=7.8 \mathrm{~Hz}) ;{ }^{13} \mathrm{C}$ (300 MHz; DMSO-d6) $\delta_{\mathrm{ppm}}: 19.2 ; 21.5$; 55.6; 55.8; 59.6; 88.3; 92.2; 114.4-157.3; 154.3; 168.5.

Elemental analysis: $\mathrm{C}_{21} \mathrm{H}_{22} \mathrm{~N}_{2} \mathrm{O}_{4}$ requires $\mathrm{C}, 68.84 ; \mathrm{H}, 6.05$; N, 7.65\%; Found: C, 68.81; H, 5.99; N, 7.59\%.

\section{6-Methoxy-3,5-di(4-methoxyphenyl)-6a-methyl- 3a,5,6,6a-tetrahydro-4H-pyrrolo[3,4-d]isoxazole- 4-one (3g)}

Yield $=80 \%$ white solid. $\mathrm{mp}=179-180{ }^{\circ} \mathrm{C}$. IR $(\mathrm{KBr})$ ymax/cm ${ }^{-1}: 1640(\mathrm{C}=\mathrm{N}) ; 1740(\mathrm{C}=0) .{ }^{1} \mathrm{H}$ NMR (300 MHz; DMSO-d6) $\delta_{\mathrm{ppm}}: 1.69$ (s, 3H, $\left.\mathrm{CH}_{3}\right) ; 3.74\left(\mathrm{~s}, 6 \mathrm{H}, \mathrm{OCH}_{3}\right) ; 3.79$ (s, 3H, $\left.\mathrm{OCH}_{3}\right) ; 4.16(\mathrm{~s}, 1 \mathrm{H}, 3 \mathrm{a}-\mathrm{H}) ; 7.17$ (s, 1H, 6-H); 6.93 $(\mathrm{d}, 2 \mathrm{H}, J=9 \mathrm{~Hz})$ and $7.38(\mathrm{~d}, 2 \mathrm{H}, J=9 \mathrm{~Hz}) ; 7.08(\mathrm{~d}, 2 \mathrm{H}$, $J=8.7 \mathrm{~Hz})$ and $7.68(\mathrm{~d}, 2 \mathrm{H}, J=8.7 \mathrm{~Hz}) \cdot{ }^{13} \mathrm{C}$ NMR $(300$ MHz; DMSO-d6) $\delta_{\mathrm{ppm}}$ : 20.5; 55.6; 55.8; 60.9; 84.8; 89.9; 114.7-161.3; 157.9; 171.2. Elemental analysis: $\mathrm{C}_{21} \mathrm{H}_{22} \mathrm{~N}_{2} \mathrm{O}_{5}$ requires $\mathrm{C}, 65.96$; $\mathrm{H}, 5.80$; N, 7.33\%; Found: $\mathrm{C}, 65.89$; H, 5.78; N, 7.37\%.

6-Methoxy-5-(4-methoxy-phenyl)-6a-methyl-3-(4-nitrophenyl)-3a,5,6,6a-tetrahydro-pyrrolo[3,4-d]isoxazol4-one (3h)

Yield $=85 \%$ white solid. $\mathrm{mp}=227-228{ }^{\circ} \mathrm{C} . \mathrm{IR}(\mathrm{KBr}) \mathrm{ymax} /$ $\mathrm{cm}^{-1}: 1645(\mathrm{C}=\mathrm{N})$; $1745(\mathrm{C}=\mathrm{O}) .{ }^{1} \mathrm{H}$ NMR (300 MHz; DMSOd6) $\delta_{\mathrm{ppm}}: 1.69\left(\mathrm{~s}, 3 \mathrm{H}, \mathrm{CH}_{3}\right) ; 3.79\left(\mathrm{~s}, 3 \mathrm{H}, \mathrm{OCH}_{3}\right) ; 4.17(\mathrm{~s}, 1 \mathrm{H}$, 3a-H); 7.23-8.03 (m, 9H, Harom). ${ }^{13} \mathrm{C}$ NMR (300 MHz; DMSO-d6) $\delta_{\text {ppm }}: 20.4 ; 55.7$; 55.8; 61.0; 84.9; 90.9; 115.1160.9; 157.8; 170.8. Elemental analysis: $\mathrm{C}_{20} \mathrm{H}_{19} \mathrm{~N}_{3} \mathrm{O}_{6}$ requires C, 60.45; H, 4.82; N, 10.57\%; Found: C, 60.40; H, 4.79; N, $10.55 \%$.

[4] Ben Hamadi, N.; Msaddek, M. Synthesis and reactivity of $\mathrm{N}$-sugar-maleimides: an access to novel highly substituted enantiopure pyrazolines. Tetrahedron: Asymmetry 2012, 23 , 1689-1693.

[5] Ben Hamadi N.; Msaddek, M. An unexpected transformation by reduction of isoxazolines. C. R. Chimie, 2012, 15, 653-657.

[6] Ben Hamadi, N.; Msaddek, M. 1,3-dipolar cycloadditions of arylnitrile oxides and 2-diazopropane with 5-hydroxy-3-methyl1,5-dihydropyrrol-2-one derivatives. C. R. Chimie, 2011, 14, 891-895.

[7] Ruige, Y.; Yuanyuan, Z.; Hui, X. Synthesis of novel isoxazoline-containing podophyllotoxin $/ 2^{\prime}\left(2^{\prime}, 6^{\prime}\right)(\mathrm{di})$ 
halogenopodophyllotoxin derivatives and their insecticidal/ acaricidal activities. Bioorganic \& Medicinal Chemistry Letters, 2018, 28, 1410-1416.

[8] Kamalneet, K.; Vinod, K.; Anil, K.S.; Girish, K.G. Isoxazoline containing natural products as anticancer agents: A review. European Journal of Medicinal Chemistry, 2014, 77, 121-133.

[9] Hajlaoui, K.; Guesmi, A.; Ben Hamadi, N.; Msaddek, M. Synthesis of carbohydrate-substituted isoxazoles and evaluation of their antitubercular activity. Heterocyclic Communications, 2017, 23, 225-229.

[10] Zhang, Y.; Hubbard, J. W.; Akhmedov, N. G.; Petersen, J. L.; Söderberg B.C.G. Total Synthesis of the Tetracyclic Indole Alkaloid Ht-13-B. J. Org. Chem. 2015, 80, 4783-4790.

[11] Zhang, Y.; McArdle, W.L.; Hubbard, J. W.; Akhmedov, N. G.; Söderberg B.C.G. Total synthesis of the tetracyclic indole alkaloid ht-13-A. Tetrahedron Lett. 2016, 57, 2865-2867.

[12] Reddy, C.R.; Radhika, L.; Kumar, T.P.; Chandrasekhar, S. First Acid-Catalyzed Entry to $O$-Alkylated Hydroximides from Benzylic Alcohols. Eur. J. Org. Chem. 2011, 5967-5970.

[13] Reddy, M.D.; Fronczek, F.R.; Watkins, E.B. Rh-Catalyzed, Regioselective, C-H Bond Functionalization: Access to Quinoline-Branched Amines and Dimer. Org. Lett. 2016, 18, 5620-5623.
[14] Reddy, M.D.; Uredi, D.; Watkins, E.B. A general method for the metal-free, regioselective, remote $\mathrm{C}-\mathrm{H}$ halogenation of 8-substituted quinolines. Chemical Science 2018, 9 , 1782-1788.

[15] Nobuyuki, M.; Toshiki, N.; Masaomi, H.; Kazuhiro, I.; Junichiro, B.; Hidemi, Y.; Kunihiko, T. Regioselective reduction of maleimide and citraconimide derivatives: general preparation of 5-hydroxy-1,5-dihydropyrrol-2-one. J. Chem. Soc. Perkin Trans. 1 2002, 707.

[16] Ben Hamadi, N.; Msaddek, M. Direct oxidation of $\Delta 2$-isoxazolines synthesis by metal ion-mediated diastereoface-selective 1,3-dipolar cycloaddition with "activated" DMSO. Arabian Journal of Chemistry, 2017, 10, s800-s803.

[17] Blake, A.J.; Forsyth, A.C.; Paton, R.M. Regio- and face-selective 1,3-dipolar cycloadditions to levoglucosenone. J. Chem. Soc. Chem. Commun. 1988, 440-442.

[18] Garcıa, R. J.L.; Fraile, A.; Gonzalez, G.; Martın, M.R.; Clemente, F.R.; Gordillo, R.R.; A New and Efficient Synthesis of Pyrrolo[2,3-d]pyrimidine Anticancer Agents: Alimta (LY231514, MTA), Homo-Alimta, TNP-351, and Some Aryl 5-Substituted Pyrrolo[2,3-d] pyrimidines. J. Org. Chem. 2003, 68, 6522-6534. 